Check for updates

Cite this: RSC Adv., 2017, 7, 35752

Received 16th June 2017

Accepted 13th July 2017

DOI: $10.1039 / \mathrm{c} 7 \mathrm{ra06744e}$

rsc.li/rsc-advances

\section{Enhancing perovskite film fluorescence by simultaneous near- and far-field effects of gold nanoparticles}

\author{
Xiaoyan Wu, (DD *a Yanglong Li, ${ }^{a}$ Lingyuan $\mathrm{Wu}{ }^{a} \mathrm{Bo} \mathrm{Fu}{ }^{a}$ Guodong Liu, ${ }^{a}$ Dayong Zhang, ${ }^{a}$ \\ Jianheng Zhao, ${ }^{a}$ Ping Chen ${ }^{\star b}$ and Linlin Liu ${ }^{\star c}$
}

In this work gold nanoparticles (Au NPs) are incorporated into a PEDOT:PSS layer, resulting in $~ 4.0$ fold enhanced fluorescence emission of a $\mathrm{CH}_{3} \mathrm{NH}_{3} \mathrm{PbBr}_{3}$ film. Through experiments and theoretical simulation, we first demonstrated that the enhancement comes from the simultaneous near- and farfield effects of Au NP enhanced perovskite fluorescence.
Organic-inorganic hybrid halide perovskites have emerged as a new class of photovoltaic materials with high efficiencies driven by the large absorbance and long-range balanced electron and hole transportation. ${ }^{1,2}$ Recently, perovskites have been making definitive strides as promising light-emitting diodes (LEDs) and for use in tuneable amplified spontaneous emission lasing. ${ }^{3-6}$ During the past two years, impressive progress has been made in perovskite LEDs (PeLEDs) and lasing devices; one of the most important tasks is increasing the photoluminescence efficiency of active layer materials, ${ }^{7,8}$ because the electroluminescence (EL) efficiency is theoretically proportional to the photoluminescence (PL) efficiency. ${ }^{9}$ Driven by this motivation, scientists not only attempted different material structures of the active layer, but also searched for ways of achieving additional photo-physical assistance during device fabrication for further enhancement. ${ }^{10-12}$

Metallic nanostructures have been widely employed in the organic optoelectronic devices for performance improvement, due to their optical and electrical effect such as metal enhanced fluorescence (MEF) ${ }_{13-19}$ energy transfer, ${ }^{20}$ electrical effect, ${ }^{21,22}$ and so on. ${ }^{23,24}$ The enhancement utilizing MEF effect usually has a close dependence on the distance between the emitters and the surface of the metallic nanoparticles (NPs). When the fluorophore molecule is brought into the proximity of the metallic NPs based substrate, two maximum enhancements would be observed as a function of the interlayer thickness, which would be originated from near-field (less than $10 \mathrm{~nm})^{14}$

${ }^{a}$ Institute of Fluid Physics, China Academy of Engineering Physics, Mianyang 621900, China.E-mail: wuxiaoyan1219@sina.cn

${ }^{b}$ School of Physical Science and Technology, MOE Key Laboratory on Luminescence and Real-Time Analysis, Southwest University, Chongqing 400715, China. E-mail: chenping206@126.com

'Institute of Polymer Optoelectronic Materials and Devices, State Key Laboratory of Luminescent Materials and Devices, South China University of Technology, Guangzhou 510640, China.E-mail: mslliull@scut.edu.cn and far-field (greater than $60 \mathrm{~nm}$ ). ${ }^{24-27}$ Recently, Balakrishnan et al. reported the enhanced PL intensity of $\mathrm{CsPbBr}_{3}$ nanocrystals by perovskites-gold (Au) hybrid structure; ${ }^{28} \mathrm{Zhang}$ et al. inserted silver nanorods into NPB layer to improve the PeLEDs performance. ${ }^{29}$ The enhancement reported above arised from near-field effect of metal NPs. Nevertheless, in theoretically with the appropriate devices structure, the near- and far-field effect of Au NPs both could overlap the emitting region of perovskites (usually with thickness of tens to hundreds nanometers). Specifically, the simultaneous utilizing near- and far-field would enhance fluorescence greater than single optical effect of metal NPs. However, as far as we know, there is very few reports focus on the dual-optical effect of metallic NPs enhance perovskites film fluorescence.

In this paper, $\sim 4.0$ fold enhanced fluorescence intensity of $\mathrm{CH}_{3} \mathrm{NH}_{3} \mathrm{PbBr}_{3}\left(\mathrm{MAPbBr}_{3}\right)$ film was achieved by doping Au NPs into the PEDOT:PSS buffer layer. We demonstrated that the enhancement originated from the simultaneous near- and farfield optical effect of Au NPs via experiments and theoretical simulation, which was also the first report that utilizing the dual near- and far-field optical effect in metal enhanced perovskites fluorescence.

The preparation of the mixed $\mathrm{MAPbBr}_{3}$ perovskites film is summarized in Scheme 1. The PEDOT:PSS was spin-coated onto

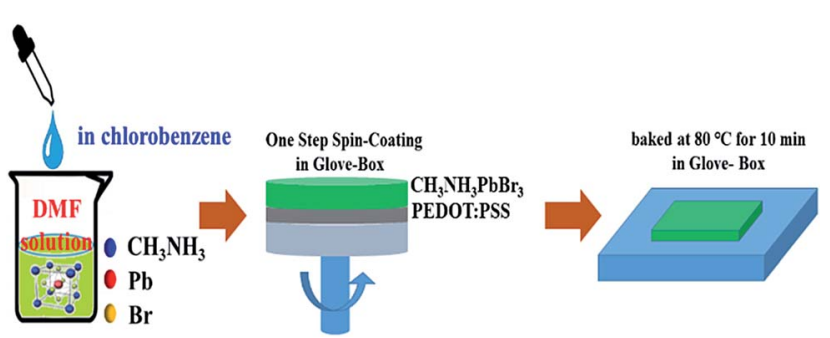

Scheme 1 The preparation of the mixed $\mathrm{MAPbBr}_{3}$ perovskites. 
the quartz substrate $(4500 \mathrm{rpm}, 40 \mathrm{~s})$ and baked at $120{ }^{\circ} \mathrm{C}$ for 20 min under ambient conditions. The $\mathrm{MAPbBr}_{3}$ solution was prepared by dissolving $\mathrm{CH}_{3} \mathrm{NH}_{3} \mathrm{Br}$ and $\mathrm{PbBr}_{2}$ (Xi'an Polymer Light Technology Corp, $\mathrm{CH}_{3} \mathrm{NH}_{3} \mathrm{Br}>99.9 \%, \mathrm{PbBr}_{2}>99.9 \%$ ) with 1.5 : 1 molar ratio in DMF (Alfa-Aesar, anhydrous) solution (the concentration ratio is based on our previous research work ${ }^{\mathbf{1 1}}$ ). Then chlorobenzene solution was added dropwise into $\mathrm{MAPbBr}_{3}$ DMF until saturation, in order to accelerate crystallization of perovskites. The precursor solution was spin-coated onto PEDOT:PSS film in the glove-box $\left(\mathrm{H}_{2} \mathrm{O}\right.$ and $\left.\mathrm{O}_{2} \leq 0.1 \mathrm{ppm}\right)$ at a speed of $4000 \mathrm{rpm}$ for $60 \mathrm{~s}$, and annealed at $80^{\circ} \mathrm{C}$ for $10 \mathrm{~min}$.

X-ray diffraction (XRD) pattern displayed in Fig. 1a gave diffraction peaks at $14.90^{\circ}, 21.08^{\circ}, 29.98^{\circ}, 33.62^{\circ}, 36.92^{\circ}, 42.94^{\circ}$ and $45.68^{\circ}$, which were assigned to the (100), (110), (200), (210), (211), (220) and (300) planes of perovskites structure, respectively. According to the previous report, ${ }^{30}$ the XRD indicated pure perovskites phase for $\mathrm{MAPbBr}_{3}$, which suggested a lamellar structure with $\mathrm{PbBr}_{2}$ 2D-layer parallel to the substrate. The inset of Fig. 1a showed the $\mathrm{MAPbBr}_{3}$ film (deposited on quartz/PEDOT:PSS substrate) with $\sim 365 \mathrm{~nm}$ UVlight irradiation. The cross-section SEM image of the device has also been given in Fig. 1b, the thicknesses of the $\mathrm{MAPbBr}_{3}$ film was $\sim 500 \mathrm{~nm}$ under the fabrication method. Top view of SEM images of the $\mathrm{MAPbBr}_{3}$ film can be seen in Fig. 1c. It exhibited a uniform and dense surface morphology with average grain size of 300-500 nm. Au NPs used here of $20 \mathrm{~nm}$ diameter size (TEM image in inset Fig. 2a) were synthesized according to the classical Frens method, ${ }^{31}$ the typical extinction $520 \mathrm{~nm}$ of the Au NPs aqueous solution was shown in Fig. 2a.
The PL spectra (with $\sim 365 \mathrm{~nm}$ excitation) and absorbance spectra of $\mathrm{MAPbBr}_{3}$ film were shown in Fig. 2a. It can be seen that the $\mathrm{MAPbBr}_{3}$ film showed a typical green emission around $\sim 534 \mathrm{~nm}$ with narrow FWHM of $\sim 24 \mathrm{~nm}$.

The maximum fluorescence intensity enhancement showed about $\sim 4.0$ fold after doping Au NPs into PEDOT:PSS under the optimized concentration $12 \mathrm{pM}$ in Fig. 2b. With the increased $\mathrm{Au}$ NPs concentration, the PL intensity increased (in inset Fig. 2b). The absorbance spectra and time-resolved PL spectra in Fig. $2 \mathrm{c}$ and d provided the direct evidence of the increased PL intensity. The absorbance intensity of $\mathrm{MAPbBr}_{3}$ film improved $45 \%$ under 12 pM concentration Au NPs doping, because of the enhanced absorbance probability (get rid of the Au NPs absorbance). ${ }^{32}$ The time-resolved PL spectra of $\mathrm{MAPbBr}_{3}$ layer on PEDOT:PSS film in Fig. 2d showed the exciton lifetime is $7.28 \mathrm{~ns}$ without Au NPs, but surprisingly, decreases to $0.31 \mathrm{~ns}$ with $\mathrm{Au}$ NPs. Therefore, time-resolved PL measurement provided the direct observation of the enhanced spontaneous emission rate of $\mathrm{MAPbBr}_{3}$ induced by Au NPs. From the inset in Fig. 2b-d, PL would be decreased until the critical point $12 \mathrm{pM}$, which maybe due to the nanoparticles aggregation at the high $\mathrm{Au}$ NPs density. ${ }^{\mathbf{1 4 1 6}}$ High aggregation Au NPs would exposed out of the PEDOT:PSS surface (top view and cross-section of SEM as shown in Fig. 3a-d), then the direct contact between $\mathrm{Au}$ NPs and $\mathrm{MAPbBr}_{3}$ film would seriously quench the fluorescence by nonradiative Fröster energy transfer. ${ }^{14,33}$

In order to explore the enhancement mechanism, we simulate the near- and far-field model around Au NPs in Fig. 4. The energy matching and distance dependence characters would be the primary difference. For near-field, when the frequency of
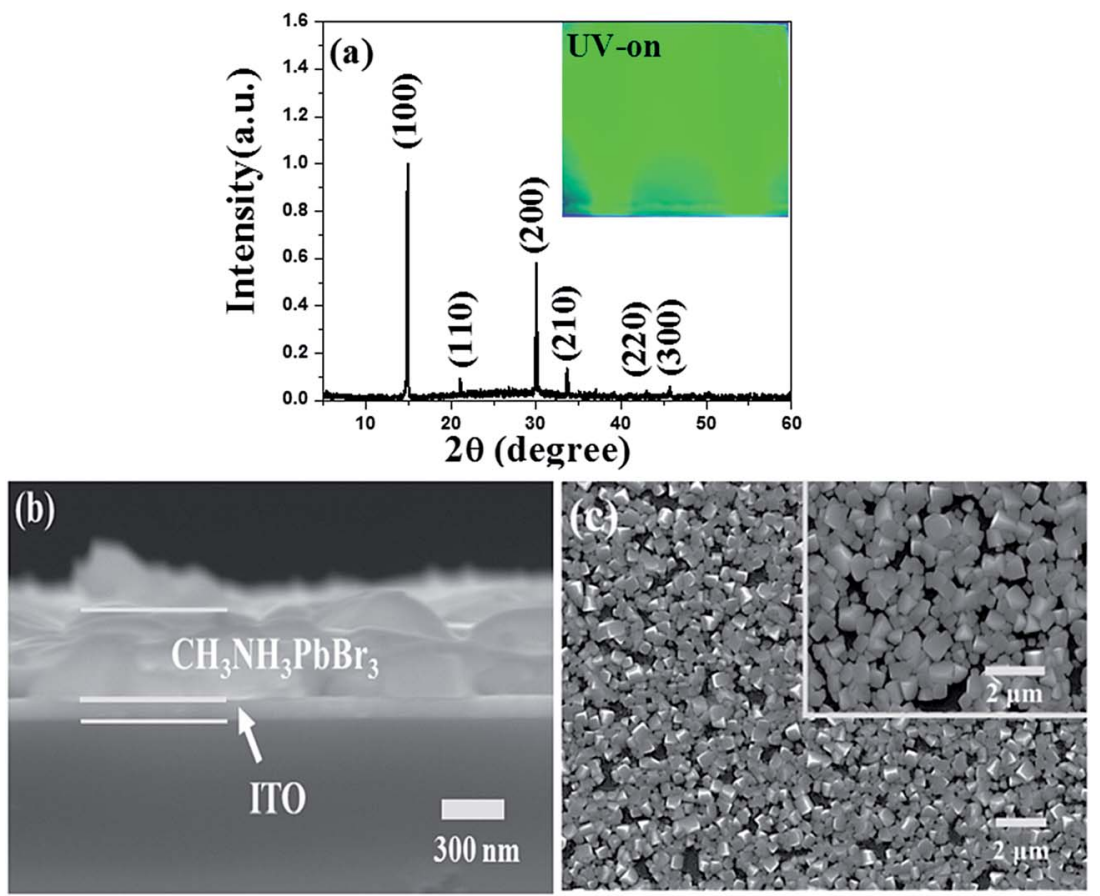

Fig. 1 (a) XRD spectra of green-emitting $\mathrm{MAPbBr}_{3}$ crystal film, inset: image of $\mathrm{MAPbBr}_{3}$ film under UV-365 nm excitation, (b) cross-section SEM and (c) SEM image of $\mathrm{MAPbBr}_{3}$ film. 

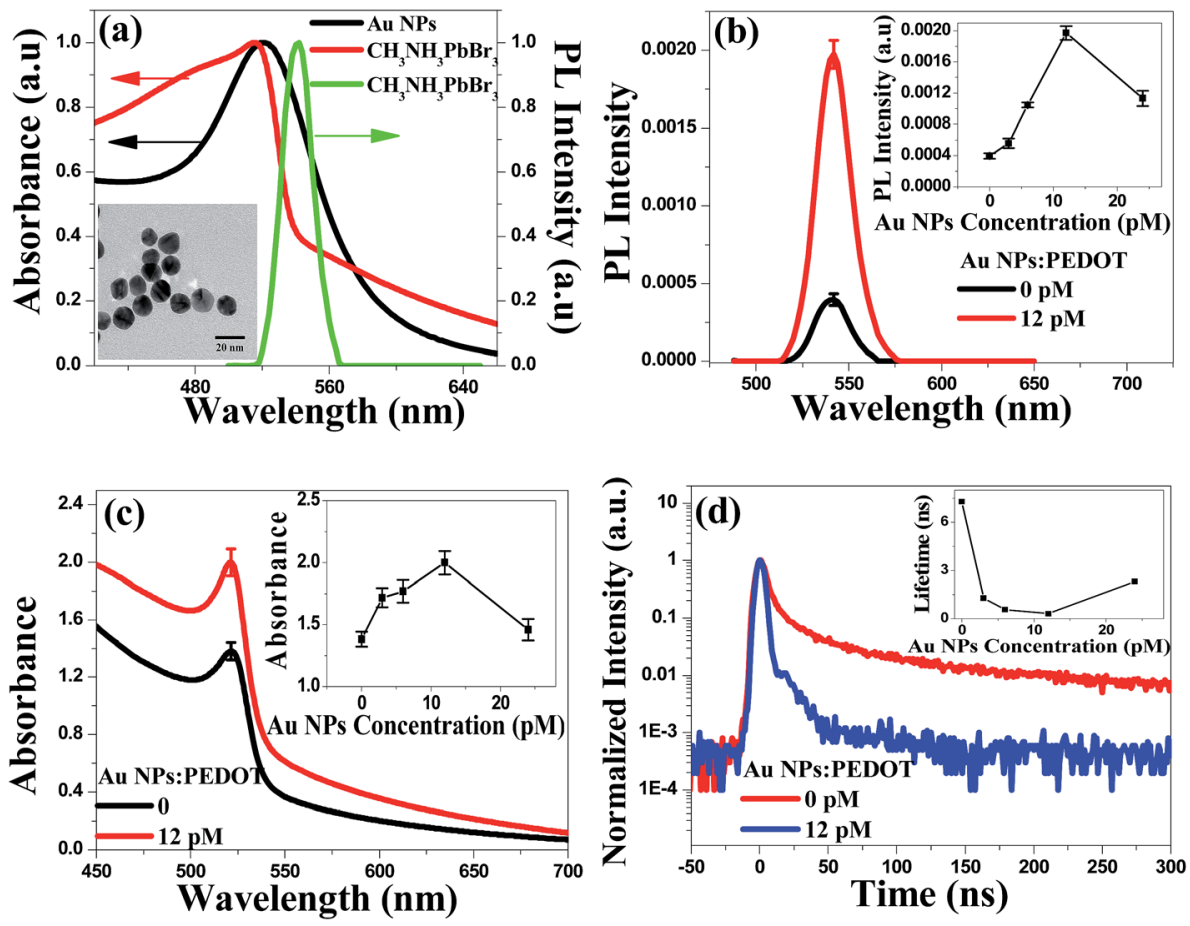

Fig. 2 (a) Photoluminescence spectra of $\mathrm{MAPbBr}_{3}$ film, absorbance spectra of $\mathrm{MAPbBr}_{3}$ film and Au NPs aqueous solution respectively, inset: TEM image of Au NPs. (b) Photoluminescence spectra, (c) UV-vis spectra, (d) time-resolved PL spectra in the presence of MAPbBr 3 film on PEDOT:PSS doping into Au NPs. The inset in $(b-d)$ is the photoluminescence, UV-vis and time-resolved PL spectra changing uptrend under different Au NPs doping ratio.
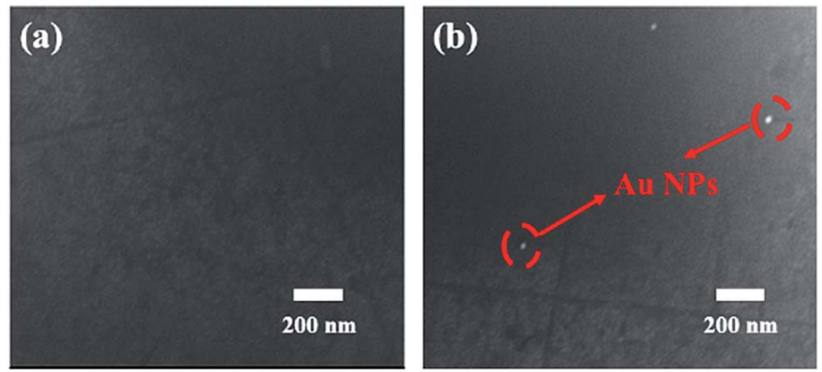

(c)

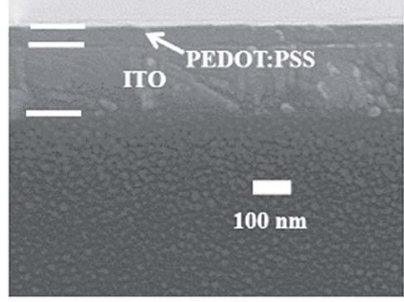

(d)

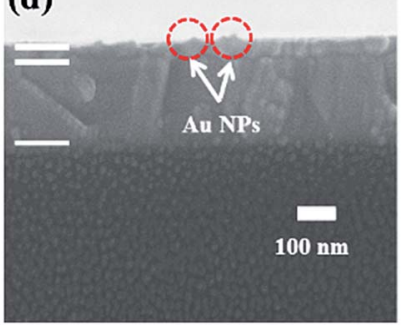

Fig. 3 Top view and cross-section of SEM image of the dispersion of Au NPs in PEDOT:PSS under (a) low, (c) none, (b and d) high concentration doping ratio.

incident light is located in the resonant frequency of metallic NPs, resonance will occur to form the near-field effect around metallic NPs in the range of 5-10 nm. Fig. 4a shows the near electric-field distribution of $20 \mathrm{~nm}$-Au NPs simulated by finitedifferent time-domain (FDTD). We can see that the electric- field intensity strongly distribute around the neighbourhood of the NPs, and working distance is in the range of 5-10 nm. The intensity of near-field degraded very quickly as a function of
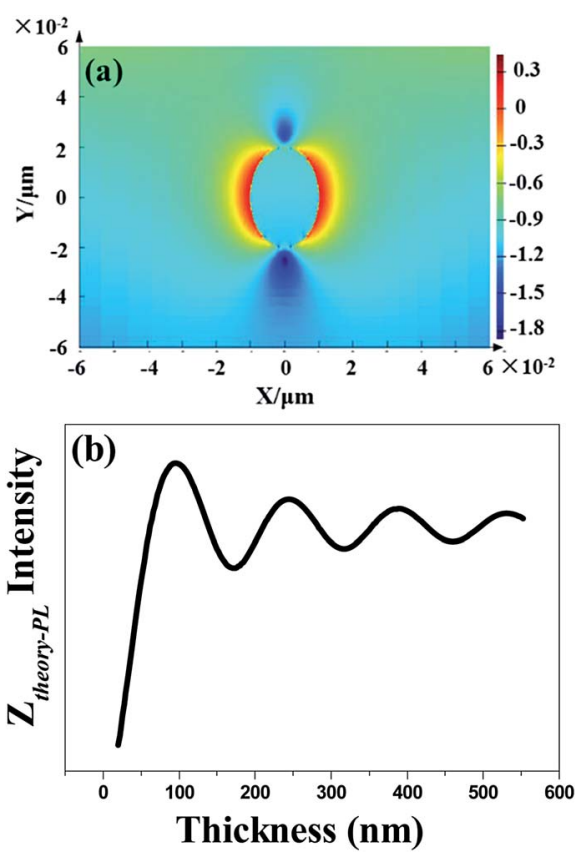

Fig. 4 Theoretical simulation of (a) near-electric field profile, (b) farfield distribution. 
distance, which is nearly disappeared when distance larger than $10 \mathrm{~nm}$. The thickness of PEDOT:PSS thin film is the key factors to determine the effective coupling between near-field and emissive layer. Thus, the thickness of PEDOT:PSS buffer layer (less than $30 \mathrm{~nm}$ ) would be thin enough to put the emitting layer into the scoped of near-field as shown in Scheme 2a.

For far-field, the enhancement ratio for respective wavelength is not related to the intrinsic frequency of metallic NPs as in near-field effect, but depends on fluorophore/metallic NPs distance $d$ and refractive index of media $n$. Thus enhancement for different wavelength would be easily achieved by distance $d$ optimization. We also established the model of far-field enhancement of $\mathrm{Au}$ NPs. Recently we demonstrated utilizing far-field of Au NPs to enhance chromophores emissive in the full-visible spectral range in organic light-emitting diodes. ${ }^{\mathbf{1 4 , 1 5 , 2 5}}$ The enhancement factor $Z_{\text {theory-PL }}$ of far-field effect is given by

$$
\begin{aligned}
Z_{\text {theory-PL }}= & q_{\mathrm{d}} / q=\left\{q+(1-q)\left[1+\frac{3}{2} q R\right.\right. \\
& \left.\left.\times\left(-\frac{\sin (\gamma-\delta)}{\gamma^{3}}+\frac{\sin (\gamma-\delta)}{\gamma}+\frac{\cos (\gamma-\delta)}{\gamma^{2}}\right)\right]^{-1}\right\}^{-1}
\end{aligned}
$$

in which $q$ and $q_{\mathrm{d}}$ is quantum yield before and after the introducing of metal NPs, $\delta$ is phase shift at the reflection, $R$ is reflectivity, $\gamma$ is arbitrary value and defined as $\gamma=4 \pi n d / \lambda$, where $n$ is refractive index of luminescence material, $\lambda$ is the emission wavelength. The enhancement factors of quantum yield as a function of distance $d$ are displayed in Fig. 4b (supposing $R=$ 1 (total reflect) and $\delta=\pi$ (idea metal mirror)). By increasing of distance, the theoretical PL enhancement $Z_{\text {theory-PL }}$ in $\mathrm{MAPbBr}_{3}$ material presents the trend: at first it would rise with the increased distance and then it would reduce after the maximum value. The far-field enhancement shows multi-enhancement peak in the thickness of emitting layer, combining with the device structure in Scheme 2a. Thus near- and far-field could both overlap the emitting layer. And our previous work demonstrated that $20 \mathrm{~nm}$-size Au NPs doping into PEDOT:PSS shows negligible effect on scattering effect. ${ }^{25}$ Compare to singleoptical enhancement effect of Au NPs, there are mainly two advantages by simultaneous utilizing near- and far-field of $\mathrm{Au}$ NPs: (1) considering the device structure (Scheme 2a), it is easy to realize dual-optical effect enhancement in perovskites light emitting devices and lasing devices; (2) the fluorescence enhancement ratio by dual-optical effect would be larger than single-optical effect.

To further deeply understand the mechanism of the effective emissive enhancement by doping Au NPs, we proposed that multiple plasmonic enhancing processes induced by dualoptical effect as shown in Scheme 2b. As shown in Scheme $2 \mathrm{~b}$, there are two main processes occur when excited: absorbance and radiative velocity. Firstly in the absorption process, the ground state electrons transit from the lowest vibrational level of $S_{0}$ to a higher vibrational level of excited state $S_{1}$ and soon return to the lowest vibrational level of $S_{1}$ by non-radiative internal conversion (IC) process. Then the excited electrons transit from the lowest vibrational level of $S_{1}$ to $S_{0}$ by radiative and non-radiative ways. $20 \mathrm{~nm}$-Au NPs were buried in the PEDOT:PSS film randomly with proper distances to the $\mathrm{MAPbBr}_{3}$ layer. Through multiple surface plasmonic effects, the absorbance probability of $\mathrm{MAPbBr}_{3}$ can be significantly enhanced, therefore more electrons transit to higher energy level $\mathrm{S}_{1}$ by electromagnetic field (combining with Fig. 2c). From the lowest vibrational level of $S_{1}$ to $S_{0}$, the addition of Au NPS induces faster radiative velocity (combining with Fig. 2d) and leads enhanced emission. Thus, the presence of Au NPs increases the population excited electrons, and enhance the radiative velocity. Based on the multiple enhancements of absorbance and emission during light excitation, the enhancement of fluorescence will be much more effective under near- and far-field together.

In a conclusion, we achieved about $\sim 4.0$ fold fluorescence enhancement through doping Au NPs into the PEDOT:PSS. Both the experimental and theoretical results have testified that it mainly attributes to multiple near- and far-field effect of Au NPs. The scope of the near- and far-field of Au NPs could both cover with the $\mathrm{MAPbBr}_{3}$ film, which means it in favour of fluorescence enhancement most. And it is also the advantage that utilizing $\mathrm{Au}$ NPs enhancing the $\mathrm{MAPbBr}_{3}$ film fluorescence emissive. Our results may offer that Au NPs constitute a feasible and effective rout for achieving high-performance perovskites light-emitting diodes and lasing devices.

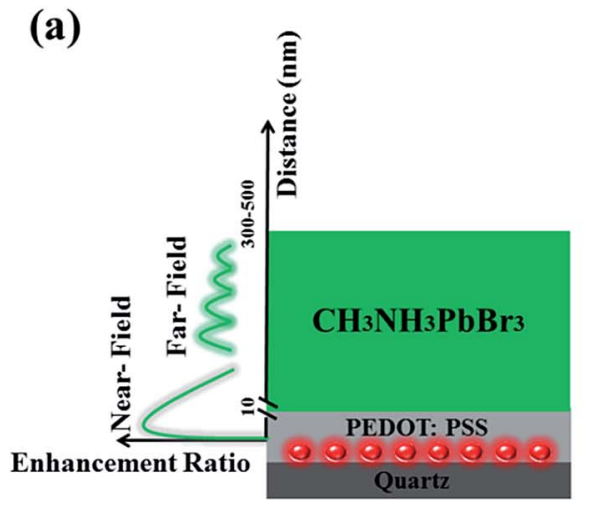

(b)

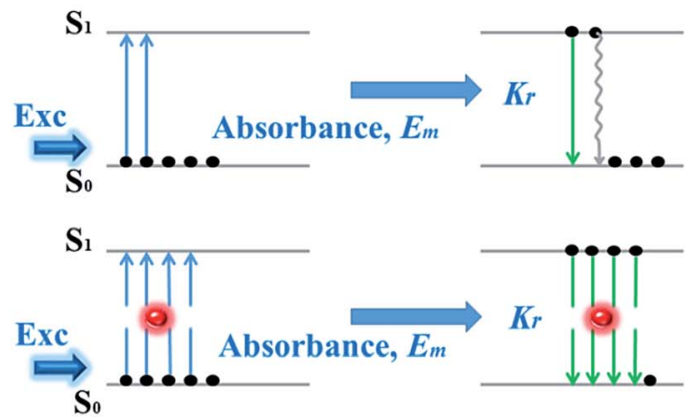

Scheme 2 (a) The near- and far-field distribution in devices, (b) diagrams of the procedure of emission without and with Au NPs. 


\section{Acknowledgements}

This work was supported by the National Natural Science Foundation of China (Grant No. 11602243, 11504300, 51473052), NSFA of China (Grant No. U1630125), Natural Science Foundation Project of CQ CSTC (Grant No. cstc2015jcyjA50002).

\section{References}

1 S. D. Stranks and H. J. Snaith, Nat. Nanotechnol., 2015, 10, 391.

2 L. K. Ono, S. H. Wang, Y. Kato, S. R. Raga and Y. B. Qi, Energy Environ. Sci., 2014, 7, 3989.

3 S. A. Velduis, P. P. Boix, N. Yantara, M. Li, T. C. Sum, N. Mathews and S. G. Mhaisalkar, Adv. Mater., 2016, 28, 6804.

4 G. Xing, N. Mathews, S. S. Lim, N. Yantara, X. Liu, D. Sabba, M. Grätzel, S. Mhaisalkar and T. C. Sum, Nat. Mater., 2014, 13, 479 .

5 Z. K. Tan, R. S. Moghaddam, M. L. Lai, P. Docampo, R. Higler, F. Deschler, M. Price, A. Sadhanala, L. M. Pazos, D. Credgington, F. Hanusch, T. Bein, H. J. Snaith and R. H. Friend, Nat. Nanotechnol., 2014, 9, 687.

6 X. F. Du, G. Wu, J. Cheng, H. Dang, K. Z. Ma, Y. W. Zhang, P. F. Tang and S. Chen, RSC Adv., 2017, 7, 10391.

7 C. Zhang, K. Wang, N. Yi, Y. Gao, M. Zhu, W. Sun, S. Liu, K. Xu, S. Xiao and Q. Song, Adv. Opt. Mater., 2016, 4, 2057.

8 R. Dhanker, A. N. Brigeman, A. V. Larsen, R. J. Stewart, J. B. Asbury and N. C. Giehink, Appl. Phys. Lett., 2014, 105, 151112.

9 X. L. Yang, G. J. Zhou and W. Y. Wong, J. Mater. Chem. C, 2014, 2, 1760.

10 Q. Liao, K. Hu, H. H. Zhang, X. D. Wang, J. N. Yao and H. B. Fu, Adv. Mater., 2015, 27, 3405.

11 P. Chen, Z. Y. Xiong, X. Y. Wu, M. Shao, X. J. Ma, Z. H. Xiong and C. H. Gao, J. Phys. Chem. Lett., 2017, 8, 1810.

12 F. Deschler, M. Price, S. Pathak, L. E. Klintberg, D. D. Jarausch, R. Higler, S. Hüttner, T. Leijtens, S. D. Stranks, H. J. Snaith, M. Atatüre, R. T. Phillips and R. H. Friend, J. Phys. Chem. Lett., 2014, 5, 1421.

13 J. H. Peng, X. J. Xu, Y. Tian, J. S. Wang, F. Tang and L. D. Li, Appl. Phys. Lett., 2014, 105, 173301.
14 X. Y. Wu, L. L. Liu, W. C. H. Choy, T. C. Yu, P. Cai, Y. J. Gu, Z. Q. Xie, Y. N. Zhang, L. Y. Du, Y. Q. Mo, S. P. Xu and Y. G. Ma, ACS Appl. Mater. Interfaces, 2014, 6, 11001.

15 X. Y. Wu, L. L. Liu, Z. C. Deng, L. Nian, W. Z. Zhang, D. H. Hu, Z. Q. Xie, Y. Q. Mo and Y. G. Ma, Part. Part. Syst. Charact., 2015, 32, 686.

16 X. Y. Wu, L. L. Liu, T. C. Yu, L. Yu, Z. Q. Xie, Y. Q. Mo, S. P. Xu and Y. G. Ma, J. Mater. Chem. C, 2013, 1, 7020.

17 Y. H. Su, Y. F. Ke, S. L. Cai and Q. Y. Yao, Light: Sci. Appl., 2012, 1, e14.

18 J. H. Peng, F. Tang, X. J. Xu, M. P. Jia and L. D. Li, RSC Adv., 2016, 6, 38148.

19 N. Y. Kim, S. H. Hong, J. W. Kang, N. S. Myoung, S. Y. Yim, S. Jung, K. Lee, C. W. Tu and S. J. Park, RSC Adv., 2015, 5, 19624.

20 J. H. Park, Y. T. Lim, O. O. Park, J. K. Kim, J. W. Yu and Y. C. Kim, Chem. Mater., 2004, 16, 688.

21 P. J. Jesuraj and K. Jeganathan, RSC Adv., 2015, 5, 684.

22 W. Y. Ji, J. Wang, Q. H. Zeng, Z. S. Su and Z. C. Sun, RSC Adv., 2013, 3, 14616.

23 M. Gu, X. P. Li and Y. Y. Cao, Light: Sci. Appl., 2014, 3, e177. 24 X. Y. Wu, L. L. Liu, Z. Q. Xie and Y. G. Ma, Chem. J. Chin. Univ., 2016, 37, 409.

25 X. Y. Wu, Y. Q. Zhuang, Z. T. Feng, X. H. Zhou, Y. Z. Yang, L. L. Liu, Z. Q. Xie, X. D. Chen and Y. G. Ma, Nano Res., 2017, DOI: 10.1007/s12274-017-1614-3.

26 J. Kummerlen, A. Leitner, H. Brunner, F. R. Aussenegg and A. Wokaun, Mol. Phys., 1993, 80, 1031.

27 C. D. Geddes and J. R. Lakowicz, J. Fluoresc., 2002, 12, 121. 28 S. K. Balakrishnan and P. V. Kamat, ACS Energy Lett., 2017, 2, 88.

29 X. Zhang, B. Xu, W. Wang, S. Liu, Y. Zheng, S. Chen, K. Wang and X. W. Sun, ACS Appl. Mater. Interfaces, 2017, 9, 4926.

30 B. Yang, X. Mao, S. Q. Yang, Y. J. Li, Y. Q. Wang, M. S. Wang, W. Q. Deng and K. L. Han, ACS Appl. Mater. Interfaces, 2016, 8, 19587.

31 G. Frens, Nat. Phys. Sci., 1973, 241, 20.

32 X. H. Zhou, L. L. Liu, X. Y. Wu, Y. Z. Yang, X. F. Jiang, X. D. Chen, Q. H. Xu, Z. Q. Xie and Y. G. Ma, J. Mater. Chem. C, 2017, 5, 1356.

33 S. A. Choulis, M. K. Mathai and V. E. Choong, Appl. Phys. Lett., 2006, 88, 213503. 